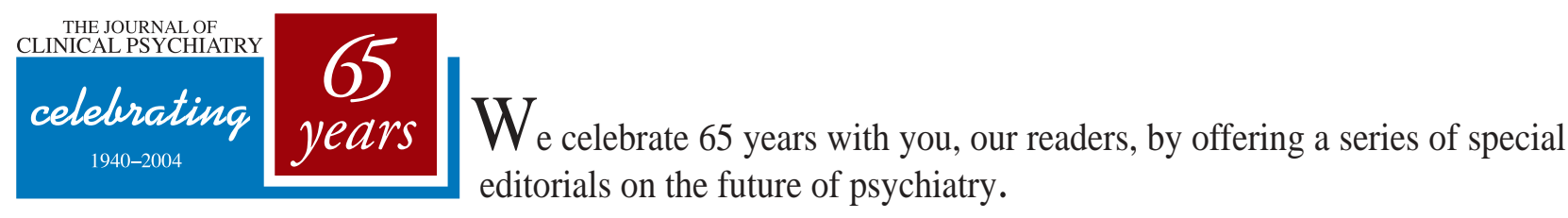

\title{
Editorial
}

\section{Medicine: The Coming Challenges and Opportunities}

\author{
Larry Culpepper, M.D., M.P.H.
}

O from the basic tally alter the shape of clinical practice by redefining our relationships with patients, redefining relationships among the health care team, redefining mechanisms of accessing and applying medical knowledge, reintegrating psychiatric and medical care, and increasing accountability for care rendered.

\section{Relationships With Patients}

Over the past 20 years, the current visit-based paradigm of practice has become increasingly dysfunctional. Given the complexity of modern life, the time- and location-bound nature of the physical visit is constraining. Instead, what patients seek is a personal, caring relationship that promotes not only access but continuity. We currently incorporate into face-to-face visits information gathering and assessment (both "initial" and "ongoing monitoring") activities, information synthesis and management decision making, and patient education, as well as the content of interpersonal therapeutic and supportive care. While face-to-face communication is optimal for some of these visit activities, for others, an asynchronous process, spread over days or weeks with repeated contacts, would be better.

Our initial steps in communicating with patients by e-mail provide a glimpse of the future. Patients might eventually interact with powerful clinical databases that in turn provide the infrastructure for clinicians to maintain a much more continuous and personal relationship with their patients. Instead of spending time inquiring

From the Department of Family Medicine, Boston Medical Center at Boston University School of Medicine, Boston, Mass.

Corresponding author and reprints: Larry Culpepper, M.D. Department of Family Medicine, Boston University School of Medicine, 1 Boston Medical Center Place, Dowling 5 South, Boston, MA 02118 (e-mail: larry.culpepper@bmc.org). about symptoms, monitoring medications, and other clinical parameters, the clinician will focus on the unique aspects of patients and their needs. Through the addition of videoconferencing and asynchronous messaging to in-person visits, clinicians will maintain a more intense involvement with their patients, an involvement not feasible in our current visit-driven system. This expansion of the modalities through which we interact with and work with our patients to make clinical decisions will challenge our current financial, legal, and ethical framework but will greatly improve our ability to help our patients. Primary care and psychiatry together will be central to redefining the doctor-patient relationship of the future.

\section{Relationships Among the Health Care Team}

Not only will new ways of communicating redefine the doctor-patient relationship, but they will also increase communication among health care providers involved with a patient. Current electronic records and messaging systems provide insight into the potential benefit of such increased teamwork. In some settings, patients are also being given direct electronic access to their records. However, electronic medical records are still only visitbased systems for archiving records of what has occurred as a prompt for future clinical interactions rather than information systems that also facilitate ongoing care. As we evolve our relationships with patients into ones that are more continuous, we will also need to redefine how the patient's primary clinician mobilizes and coordinates other expertise to optimize patient care. This evolution has the potential to decrease the professional isolation inherent in clinical practice while minimizing the current error-prone confusion of multiple clinicians giving uncoordinated treatment recommendations to patients.

\section{Mechanisms of Accessing and Applying Medical Knowledge}

Recent decades have seen an explosion in medical research and publication. They also have seen the devel- 
opment of powerful tools to search for information, the development of methods and statistical procedures for analyzing and synthesizing such information, and the development of standards for evaluating the merits of applying emerging evidence to patient care. However, to date, these steps are generally divorced from the specific patient information.

The emergence of electronic record systems and information search systems holds potential for coupling new knowledge with specific patients. The new methods available for clinicians to use in their interactions with patients will most likely help clinicians make individualizing their evidence-based "best practice" recommendations a priority. In both primary care and psychiatric relationships, it will require the clinician's understanding of the person behind the patient.

\section{Reintegrating Psychiatric and Medical Care}

One of the most exciting breakthroughs of recent decades is the evolving understanding that much of psychiatry involves care of patients who in reality have diseases of the brain, and that such pathology not only impacts human behavior but also contributes directly and indirectly to other diseases. Our realization that depression doubles the likelihood of initially developing cardiovascular disease, diabetes, and other chronic medical illnesses suggests that mental health care is fundamental to efforts to prevent chronic medical illness. That depression dramati- cally increases rates of mortality and complications in a wide range of patients once they have developed medical diseases (e.g., cardiac, post-stroke, HIV/AIDS, and diabetes) also suggests that care for mental illness will become an essential component of the care of patients with these diseases. Given the emergence of highly effective medication and other treatment strategies for psychiatric illnesses, it is now possible for such treatments to be an important component of chronic medical disease care. However, such integrated care strategies require involved health care professionals to communicate and coordinate their care plans.

\section{Accountability for Care Rendered}

Financial inflation in the medical system, increased recognition of medical errors, and high expectations of emerging treatments are among the pressures forcing an increased accountability in medical practice. These pressures will drive health care systems to institute many of the strategies that emerge from the changes outlined above, making possible the redefinition of medical care.

Together, these developments will redefine the practice of medicine. Not only will patients benefit, but clinicians will be more connected to their patients and involved in collaboration with other health professionals than in the past. These developments also will stimulate a return to the pre-Descartes integration of the mental and the physical in unified professional caring relationships. 